\title{
Investigation and Research on the Psychological Status of Children and Adolescents During the Epidemic Period of COVID-19
}

\author{
Hongping Zhong, Juanjuan Wang, Xuemei Wang, Cuicui Wang* \\ Department of Pediatrics, Yan'an University Affiliated Hospital , Yan'an, 716000 \\ Funding: Supported by Project Science and Technology Plan Project in Yan'an City, No. SL2020ZCSY-004
}

\begin{abstract}
[Abstract] Objective: The paper was to analyze the investigation and study of the psychological status of children and adolescents during the epidemic period of COVID-19. Methods: From March 5th to 11th, 2020, 1766 students from 8 to 18 years old in Yan'an area were taken as the research objects, and the psychological characteristics of this group of people during the epidemic period of COVID-19 were analyzed by the online questionnaires. Results: In the questionnaire, all children and adolescents were in good psychological conditions, and they had not shown serious negative psychological emotions, and they attached great importance to COVID-19. Conclusion: The psychological changes of children and adolescents during the epidemic period of COVID-19 are diverse. Most children and adolescents have a good mental state, and a few have negative psychological emotions. It can strengthen the psychological management of children and adolescents during the epidemic, and promote the healthy growth of children and adolescents clinically.
\end{abstract}

Key words: Epidemic period of COVID-19; Children; Adolescents; Psychological survey

Publication date: May, 2021; Publication online: 31 May, 2021

*Corresponding author: Cuicui Wang,695765898@qq.com

\section{Introduction}

In mid-December 2019, a number of patients with unexplained pneumonia with a history of exposure to the South China Seafood Market were found in Wuhan, Hubei Province, and they were quickly confirmed as a new type of coronavirus infection. As the epidemic enters its peak stage and pathogenic testing is carried out, reported cases of child infection are gradually increasing, and cases of neonatal infection are emerging ${ }^{[1]}$. During this special period, parents of children and adolescents should maintain emotional stability and mental health. Parents should maintain an optimistic attitude to avoid negative emotions in the family. Parents should pay attention to guiding children to develop an optimistic, cheerful and tolerant character, cultivate children's love of labor, sports and good interpersonal communication habits, maintain a good mental outlook, and work together to fight against COVID-19. The analysis of the psychological status of children and adolescents, and the psychological intervention of children and adolescents with unhealthy psychological emotions are related to their physical and mental development ${ }^{[2]}$. In this regard, this study conducted an investigation and analysis of the psychological conditions of children and adolescents during the epidemic period of COVID-19, which was shown below for details.

\section{General information and methods}

\subsection{General information}

The 1766 adolescents and children surveyed in Yan'an area from March 5 to 11, 2020 were the research objects. Among them, there were 890 females and 876 males. The oldest age was 18 years old, the youngest age was 8 years old, with an average of $14.6 \pm 1.1$ years old. There were 1360 urban residents, 248 town residents, and 158 rural residents. All the research objects filled out the general survey form under the supervision of their parents, and all children and adolescents 
were informed of the research content and voluntarily joined in the survey.

\subsection{Methods}

All children and adolescents were investigated by an independent questionnaire, which included the level of understanding of COVID-19, the infection probability of COVID-19, whether the health status is reported according to the regulations, whether they are worried about people coughing around them, whether they are nervous about the epidemic, whether they are worried about people returning from Hubei, whether they are affected by the negative emotions of their parents, whether they are worried about the impact of the epidemic on their studies, whether they are worried about their own situation because they can't go out to party, whether they are worried about infection because their parents are out, changes in mood and temper after the epidemic, the impact of the epidemic on sleep and emotional changes, and whether they value this epidemic.

\subsection{Observation indicators}

The psychological characteristics of this group of people during the epidemic period of COVID-19 were analyzed.

\section{Results}

Analysis of the investigation results of the psychological status of children and adolescents during the epidemic period of COVID-19

The research showed that the number of cases with a good understanding of COVID-19 and the epidemic situation were 179,1127 , and 460, respectively. The number of cases that were considered to be impossible, unlikely, and possible to be infected with COVID-19 were 818, 796, 796, and 152 cases respectively. The number of cases that reported health conditions according to regulations and reported health conditions on occasion were 1609 and 157 respectively. If there were people with cough and fever around, the number of cases that do not, occasionally, and will be nervous were 311,445 , and 1010 , respectively. The numbers of people who would not, occasionally, and worry about the people coming from Hubei were 116, 209, and 1441, respectively. The number of cases that were not, occasionally, and affected by the negative emotions of the parents were 993, 538, and 235. 339, 381, and 1046 cases did not, occasionally, and would worry about the affect of normal learning. Due to the inability to go out to the party, 850, 507, and 409 cases did not, occasionally, and did not feel worried. 840, 783, and 148 cases did not, in a small part of the time, and in quite a lot of time felt that they were in danger. 265, 508, and 993 cases did not, occasionally do, and would worry about their parents being infected due to shopping out. 959, 778, and 29 cases were unhappy, unchanged, and happier due to COVID-19. 1199, 374, and 193 cases had no change in temper, became irritable and mild after the outbreak of COVID-19. There were 1345, 195, 130, and 96 cases of no sleep effect, difficulty falling asleep, awakening from night dreams, and insomnia. 1210, 516, and 40 cases showed no change, depression, or excitement after the outbreak of COVID-19. 1733 and 33 cases were uncertain and confident of defeating the epidemic. 144, 743, 473, 406 cases did not, in a small part of the time, in quite a lot of time, always take the epidemic seriously.

\section{Discussion}

COVID-19 is a pneumonia caused by a new type of coronavirus infection that occurred in 2019. Its virus has a strong infectious ability and a high fatality rate after infection. It has a large-scale outbreak in a short period of time, which has seriously affected the daily life and health of residents $^{[3]}$. At present, it has been clinically found that COVID-19 is highly infectious and contagious. And due to the particularity of the type of virus,there is no clinically effective drug treatment, which leads to the low therapeutic effect of this type of patients. Some patients still cannot restore normal body functions after treatment, which has a serious impact on the patients' future lives ${ }^{[4]}$. National and local academic institutions attach great importance to this incident. Under the guidance and organization of the National Respiratory Diseases Clinical Research Center, the National Children's Medical Center and the Pediatrics Branch of the Chinese Medical Association, experts in pediatric respiratory, infection and critical illness have summarized and interpreted the key issues that parents care about in the protection of children from the infection of COVID-19, in order to answer the doubts of parents and eliminate unnecessary panic ${ }^{[5]}$. Studies believe that since the outbreak of COVID-19, with the increase in the number of infections in children and adolescents, the population is generally susceptible, so the country has adopted home isolation measures. Prolonged home isolation and major public health emergencies have had a certain degree of impact on the mental health of children and adolescents. Therefore, it is necessary to do a good job in the mental health of children and adolescents while doing relevant 
health protection ${ }^{[6]}$. According to research, students of different genders have different negative psychological emotions. Generally, girls' personalities are more delicate and sensitive than boys, and they are more likely to have negative psychological emotions such as worry and panic when encountering major emergencies. According to statistics, the proportion of girls, children and adolescents who have negative psychological emotions such as anxiety is 2.5 times that of boys, and there are significant differences in their anxiety symptoms ${ }^{[7]}$. In addition, children and adolescents have limited ability to analyze comprehensive problems. Girls are more inclined to actively ask and seek help when they have problems. During home quarantine, the channels for inquiries and help were blocked, which hindered the students' access to information related to the epidemic, and to a certain extent aggravated the students' negative psychological emotions ${ }^{[8]}$. What's more, during the epidemic, due to the sudden occurrence of COVID-19, China has no specific medicine to treat it. In the early stage of the epidemic, the prevalence and mortality were high, and there was no vaccine to help prevent the disease, which increased the students' fear of the epidemic, and the increased anxiety of the students' family members also affected the negative psychology of the students to a certain extent. Students who have a little knowledge of the epidemic often feel that they are in danger at any time and facing the state of death, which increases the negative psychological emotions in the students ${ }^{[9]}$. If the low and depressed mood lasts for too long, it may lead to disturbances in the regularity of life, such as loss or rapid increase of appetite, significant fluctuations in weight, and insomnia or excessive sleep for several days. Long-term depression may also cause a decline in the body's immunity. During this special period, children's parents should maintain emotional stability and mental health, maintain an optimistic attitude, and do not pass on their bad emotions to their children. Parents should pay attention to guiding their children to develop an optimistic, cheerful and tolerant character, cultivate children's love of labor, sports, and good interpersonal communication habits, and maintain a good mental outlook. Parents should also pay attention to the communication skills with their children. Listening more, admiring more, and patience are important to improve human immunity, which can prevent the virus from invading the human body and work together to fight the epidemic.

This study conducted a survey and analysis on the psychological conditions of children and adolescents during the epidemic period of COVID-19. The survey results show that in this questionnaire for children and adolescents, it can be seen that due to the popularization of COVID-19 knowledge and the implementation of epidemic prevention measures, most children and adolescents are currently in good psychological conditions and do not have serious negative psychological emotions and pay a lot of attention to COVID-19.

To sum up, the psychological changes of children and adolescents during the epidemic of COVID-19 are diverse. Most children and adolescents have good mental states, and there are a few negative psychological emotions. Clinically, it can strengthen the psychological management of children and adolescents during the epidemic, to promote the healthy growth of children and adolescents.

\section{References}

[1] Li SW, Wang Y, Yang YY, et al. Analysis of influencing factors of anxiety and emotional disorder in children and adolescents isolated at home during the epidemic of COVID-19 [J]. Chinese Journal of Child Health Care, 2020.28.214(04):53-56.

[2] Qiu QW, Zhang HY, Huang B, et al. Investigation of college students' anxiety and its influencing factors during the epidemic of COVID-19 [J]. South China Preventive Medicine, 2020,46(04):50-53.

[3] Xu Y, Zhao M, Tang XL, et al. Research on the correlation between mental health and coping styles of medical staff during the epidemic of new coronavirus pneumonia $[\mathrm{J}]$. Anhui Medicine, 2020, 041(004):368-371.

[4] Feng H, Zhe Y, Du CY, et al. Study on the physical and mental status and influencing factors of children's parents during COVID-19 [J]. Chinese Journal of Maternal and Child Health Research, 2020.31.178(02):21-26.

[5] Chen Y, Huang MY, Jin YL, et al. Investigation and analysis of psychological stress response and behavior changes of medical staff during the epidemic period of COVID-19 [J]. Journal of Wannan Medical College, 2020.39.191(06):74-78.

[6] Sun QM, Qin QS, Chen BX, et al. Investigation of psychological stress, depression, anxiety and insomnia among people in non-Hubei area during the epidemic period of COVID-19 [J]. Chinese Medical Journal, 
2020, 100(43): 3419-3424.

[7] Li Q, Wei J, Wu Q, et al. Investigation and analysis of anxiety and depression status of 183 medical staff during the epidemic period of COVID-19 [J]. Chinese Journal of Occupational Diseases, 2020, 38(12):908-911.

[8] Luo M, Jiang Y, Liu ZY, et al. Investigation and analysis of general public anxiety and depression under
COVID-19 [J]. Chinese Disaster Rescue Medicine, 2020, Volume 8, Issue 9, Pages 481-484, 2020.

[9] Zhang ZN, Liu PZ, Yu SZ, et al. Investigation and intervention measures on the psychological status of front-line nursing staff during the epidemic of COVID-19 [J]. Nursing Practice and Research, 2020.17(07):17-20. 